\title{
Life, but not as we know it
}

\author{
There are many unanswered questions regarding how the biomolecules and biomechanical processes that \\ define life came to be. A collection of Articles in this issue show how intermediates in RNA synthesis might \\ have formed and how the initiation and evolution of RNA replication might have occurred.
}

Living systems are perhaps the most fascinating examples of chemistry in action. Molecules assemble into biomachinery that can carry out complex tasks, making life possible. But how did it all begin?

This question can be tackled from a geological perspective by surveying the possible conditions under which key biomolecules might have emerged on early Earth. Alternatively, a systems-biology approach can be used to understand what biochemical processes are critical for life so that a 'retrobiosynthetic pathway' can be developed. There is only so much we can learn, however, from the highly evolved and interdependent processes that dictate contemporary biology ${ }^{1}$. Despite the limitations of these contrasting approaches, they offer chemists numerous opportunities to gain insight not only into how biomolecules might have arisen from the primordial soup, but also into how they may have come to work together to develop into primitive organisms.

In the Article on page 303 of this issue, a team led by John Sutherland demonstrate how ribose aminooxazoline possibly an important intermediate in prebiotic synthesis - can be used to make ribonucleosides and their phosphate derivatives. Earlier work by Sanchez and Orgel had identified a synthetic route from ribose to pyrimidine $\beta$-ribonucleosides via the aminooxazoline, but a low-yielding and unselective photoanomerization step was necessary to give products with the correct chirality ${ }^{2}$ - raising questions about the prebiotic plausibility of this pathway ${ }^{3}$. While pursuing improved methods for making ribose aminooxazoline using hydrosulfide, Sutherland and co-workers investigated what role such sulfur species may play in converting these intermediates into ribonucleosides. They found that the $\alpha$-thioribocytidine generated in two steps from the aminooxazoline underwent photoanomerization smoothly to form the $\beta$-anomer, which could then be transformed into the desired pyrimidine $\beta$-ribonucleoside phosphate. Finally connecting pyrimidine $\beta$-ribonucleosides to ribose - through an aminooxazoline intermediate - in a prebiotically plausible manner fills in a considerable gap in knowledge regarding the origin of RNA.

Many modern biochemical processes use enzymes to catalyse high-energy transformations but, by definition, enzymes did not exist in prebiotic media. The Article on page 310 of this issue from Matthew Powner and colleagues looks at how phosphoenol pyruvate - the highestenergy phosphate found in living systems and a key intermediate in the synthesis of nucleotides and other biomolecules - could be synthesized under prebiotic conditions. Although pyruvate has been synthesized at elevated temperatures and pressures ${ }^{4}$, these conditions - which were required to overcome the barrier to forming the phosphoenol pyruvate intermediate - are inconsistent with prebiotic models. Now, Powner and co-workers demonstrate a route that starts with the phosphorylation of glyceraldehyde, which then dehydrates to give phosphoenol pyruvaldehyde. Oxidation with manganese dioxide under ambient conditions gives phosphoenol pyruvate, suggesting that Earth-abundant metal catalysts may have been important for making high-energy compounds before enzymes existed.

Shifting away from the prebiotically plausible synthesis of small biomolecules, two other Articles in this issue are concerned with understanding how the biochemical processes that utilize these compounds first emerged. Nicholas Hud and co-workers tackle 'strand inhibition' - a process that halts DNA and RNA replication in which the template strands form a duplex, preventing nucleotide monomers from assembling on them (page 318). Although strand inhibition can be overcome at elevated temperatures ${ }^{5}$, these conditions are again inconsistent with early Earth scenarios. Seeking a suitable alternative, Hud and colleagues found that highly viscous solvents can slow the formation of template duplexes long enough to enable nucleotide monomers to assemble into gene-length strands along the single-stranded templates. It is suggested that early RNA and DNA replication may have relied on day/night cycles wherein temperature differences resulted in different levels of solvent evaporation - and therefore viscosity — leading to more separated template strands (and hence replication) during warmer days, and formation of template duplexes (and diffusion of monomers and short oligomers) when it was colder at night.

Finally, Philipp Holliger and co-workers show how biomolecules can augment and tune the activity of one another in the formation of new biological systems (page 325). The cooperation between RNA and peptides is well-known and is probably part of how the two classes of molecules evolved alongside one another. Noting that RNA polymerase ribozymes (RPR) typically require high concentrations of $\mathrm{Mg}^{2+}$ for suitable activity, but that $\mathrm{Mg}^{2+}$ also accelerates RNA degradation, Holliger and colleagues wondered how early protocells could form considering the contrasting effects of magnesium salts on these vital components. Now, the dependence of RPR on $\mathrm{Mg}^{2+}$ has been shown to be substantially lower in the presence of simple peptides, suggesting that the evolution of peptides may have accelerated the evolution of RNA. This could have permitted compartmentalization in protocells, a key prerequisite for the development of early life.

The past decade has seen advances in our understanding of what constitutes 'prebiotic plausibility' and how early biomolecules might have catalysed the formation of modern biomechanical machinery? These achievements have not only led to a better understanding of the chemical origins of life, but also represent important developments in synthesis, supramolecular chemistry and chemical biology. Although the (bio)chemistry of the early Earth will probably remain an incompletely answered question, studying the origins of life at the molecular level offers clues about our long-distant past and may result in new discoveries that could shape our future.

\footnotetext{
References

1. Sutherland, J. D. Angew. Chem. Int. Ed. 55, 104-121 (2015).

2. Sanchez, R. A. \& Orgel, L. E. J. Mol. Biol. 47, 531-543 (1970).

3. Powner, M. W. et al. ChemBioChem 8, 1170-1179 (2007).

4. Cody, G. D. et al. Science 289, 1337-1340 (2000).

5. Grossmann, T. N., Strohbach, A. \& Seitz, O. ChemBioChem 9, 2185-2192 (2008).

6. $\mathrm{Hu}$, Q. et al. Nature 534, 241-245 (2016).

7. Taylor, A. I. et al. Nature 518, 427-432 (2014).
} 